\title{
Nonlinear optical properties of synthesized porous nanostructures PMMA via template-directed methods
}

\section{Zainab F. Al-Bawi, Rawwa A. Faris, Dawood O.Altaify}

Institute of Laser for Postgraduate Studies, Baghdad University, Baghdad, Iraq

E-mail: zainabawi@yahoo.com

\begin{abstract}
crystals (opals) made of close-packed

Colloidal

polymethylmethacrylate (PMMA) were fabricated and grown by ordered periodicity and interconnected pore systems to manufacture photonic crystals. Opals were made from aqueous suspensions of monodisperse PMMA spheres with diameters between 280 and 415 nm. SEM confirmed the PMMA spheres crystallized uniformly in a face-centered cubic (FCC) array. Optical properties of synthesized pores PMMA were characterized by UV-Visible spectroscopy. It shows that the colloidal crystals possess pseudo photonic band gaps in the visible region. A combination of Bragg's law of diffraction and Snell's law of refraction were used to calculate the sphere diameter. Finally, colloidal crystals were subjected to Z-scan experiment under pulsed Q-switched Nd:YAG laser illumination to characterize it for third order nonlinear optical properties. Z-scan results show the change in transmittance of a beam, and the nonlinear refractive index is $n_{2}=9.82787 \times 10^{-12}\left(\mathrm{~cm}^{2} / G W\right)$, while the nonlinear absorption coefficient $\beta=0.04673908(\mathrm{~cm} / G W)$. These results were attributed to enhance the self-focusing arising from Kerr effect and the twophoton absorption.
\end{abstract}

Key words Z-scan, template, PMMA.

\section{Article info.}

Received: Jun. 2015

Accepted: Jun. 2015

Published: Dec. 2015

$$
\begin{aligned}
& \text { الخواص البصرية اللاخطية للبولي ميثل ميثاكريلايت النانوي المسامي المصنع بواسطة طرق }
\end{aligned}
$$

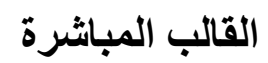

$$
\begin{aligned}
& \text { زينب فاضل مهري، رواء أحمد فارس، داود عبيد الطيفي }
\end{aligned}
$$

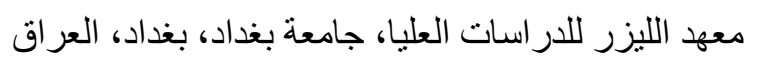




\section{Introduction}

Fabrication of porous nanostructures photonic crystals with different shapes and sizes has stimulated much interest in the last decade. Colloidal spheres with nanometer diameters are often used as building blocks for the formation porous material like photonic crystals with variable chemical composition.

Materials with high degree of porosity have broad applications in many areas including photonics, drug delivery, sensing, photocatalysis, photovoltaic in water splitting and dye sensitized solar cells $[1,2]$.

Nanostructure photonic crystals are promising materials due to their high specific surface area, enhancing charge separation, faster charge transport, visible light absorption, high interconnected porosity and unique photonic light scattering properties [3].

Colloidal spheres with nanometer diameters can be synthesized from organic or inorganic materials. The most studied and best established examples of colloidal spheres as the building blocks for fabricating porous materials are inorganic silica $\left(\mathrm{SiO}_{2}\right)$ and polymer latexes such as poly (methyl methacrylate) PMMA and poly styrene (PS) $[4,5]$.

Many Template-Directed (selfassembly) techniques have been introduced over the years for the production of colloidal crystals including; Gravity sedimentation, centrifugation, vacuum infiltration, horizontal deposition, vertical deposition via dip drawing, vertical deposition via evaporation, spin coating physical confinement, modified floating approach and exterior fields including electric and magnetic-field directed selfassemblies[6].

The most important features of photonic crystals are periodicity. Periodicity affects the propagation of electromagnetic waves in the material due to Bragg diffractions on lattice planes. They can also act as waveguides to propagate specific wavelengths in certain directions. These properties can be utilized in telecommunication systems, LEDs, semiconductor diode lasers and enhancing the performance of optoelectronics.

Nonlinear optical (NLO) materials play a major role as active components in a large range of applications such as optical communications, optical storage, optical computing, harmonic light generation, optical power limiting, optical rectifying devices, displays, printers, dynamic holography, frequency mixing and optical switching [7]. The majority of the early nonlinear optical (NLO) materials were based on inorganic crystals but in the last three decades the focus has shifted toward organic compounds due to their promising potential applications in optical signal processing [8].

In the present work we addressed the results to investigate the linear and nonlinear optical properties of colloidal crystals PMMA using the different self-assembly methods.

\section{Experimental \\ Materials and synthesis}

In our research, colloidal spheres with nanomaterial diameters suspension of PMMA were used. Three strategies have been used for the creation of colloidal crystals, the vertical deposition via evaporation, a modified floating approach and spin coating techniques

1- PMMA colloidal crystal fabrication via vertical deposition.

In the current study, suspension of nanoparticles colloidal PMMA spheres $100 \mathrm{ml},(1-10) \mathrm{wt} \%$ in $\mathrm{H}_{2} \mathrm{O}$ was used 
for the preparation of colloidal crystal templates. It ultrasonicated for $1 \mathrm{hr}$ to break up any agglomerated particles. A glass sheet was then etched for 1 hour in sodium hydroxide solution to increase its hydrophilicity for better PMMA sphere attachment and washed thoroughly with De-Ionized water several times. Fig.1 shows a vertical deposition via evaporation set-up. The beaker with glass slide and PMMA spheres suspension in it placed in an oven. After several attempts, the better temperature is at $85^{\circ} \mathrm{C}$ temperature for 48 hours. The water in the beaker had evaporated leaving a white film of the closed-packed PMMA colloidal crystal on the glass slide. Film thickness could be varied by changing the concentration of PMMA in the colloidal suspension.

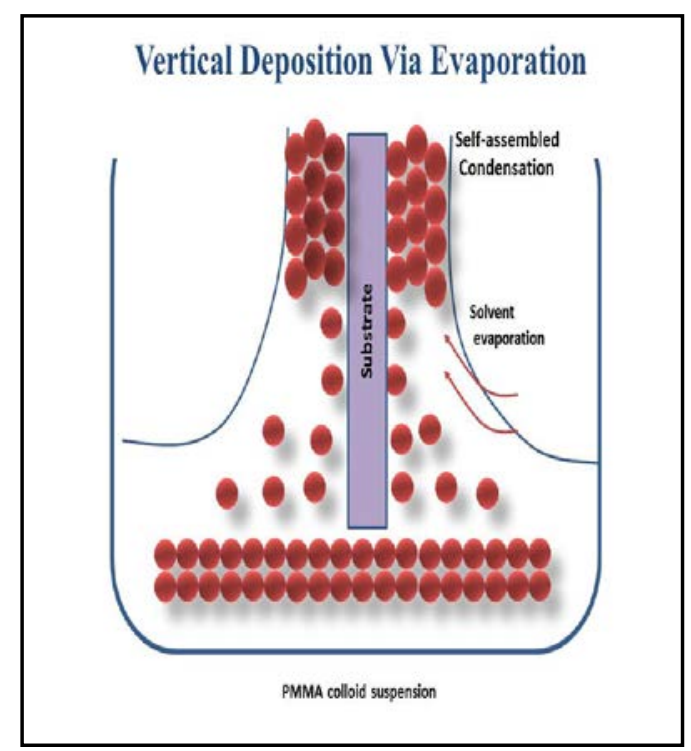

Fig.1: Vertical deposition via evaporation.

2- PMMA Colloidal Crystal Fabrication via Floating Approach PMMA spheres solution $(2 \mathrm{ml})$ was mixed with ethanol $(2 \mathrm{ml})$ in a small vial (producing a 1:1 ratio by volume). A glass sheet was cleaned twice by ultra-sonication for 10 minutes in acetone and DI water respectively. The glass was then placed in the center of a Petri dish. DI water was poured into the Petri dish, covering the sides of the glass without covering its surface. A drop of the PMMA/ethanol solution was pipetted onto the center of the glass. This was driven by the differences in surface energy between the ethanol suspension and the water in the Petri dish. More water was then carefully added to the Petri dish to rise the water level to pick up the PMMA onto a substrate. This arrangement can be shown in Fig.2.

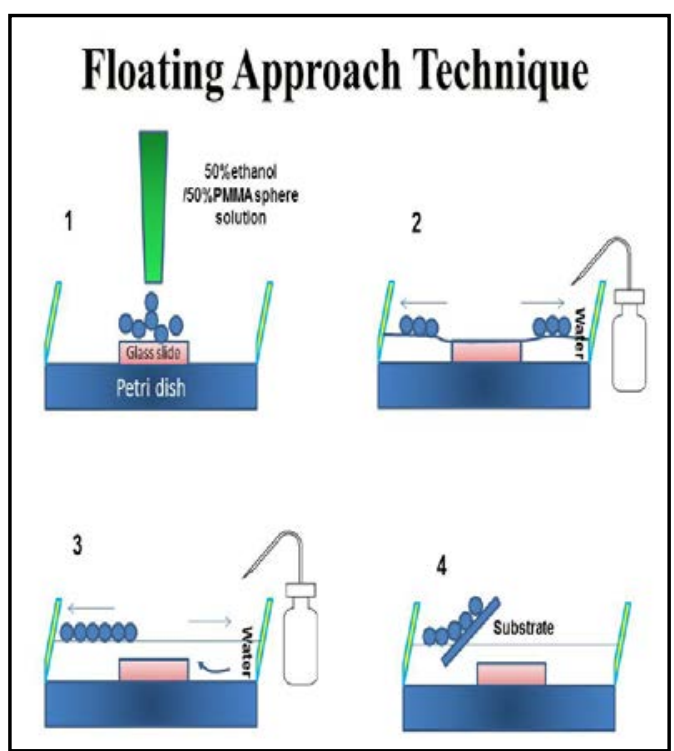

Fig.2: Floating approach technique.

3- PMMA colloidal crystal fabrication via spin coating technique

In our research, spin coating is a method used to deposit a single nanoparticles PMMA colloidal crystal on the center of flat glass substrates. The glass substrate was then rotated at two speeds low speed at certain time in order to spread the coating material by centrifugal force and high speed at certain time until the desired thickness of the film is achieved. The applied solvent is usually volatile, and simultaneously evaporates.

\section{Characterization}

UV-Vis spectroscopy was carried out to measure diffusive reflectance of colloidal spheres PMMA using an ISPREF integrating sphere coupled with a 
fiber optic cable to an ASEQ LR1 broad-range spectrometer in this work. All the SEM images in this work have been taken using a JEOL JSM-820 SEM.

Z-scan technique in which Qswitched Nd:YAG laser was used to study nonlinear properties.

\section{Results and discussions}

As it can be observed in Fig.3, the SEM image of vertical deposition via evaporation sample shows high levels of ordering with close-packed structures than other images of floating as in Fig.4 and spin coating as in Fig.5.

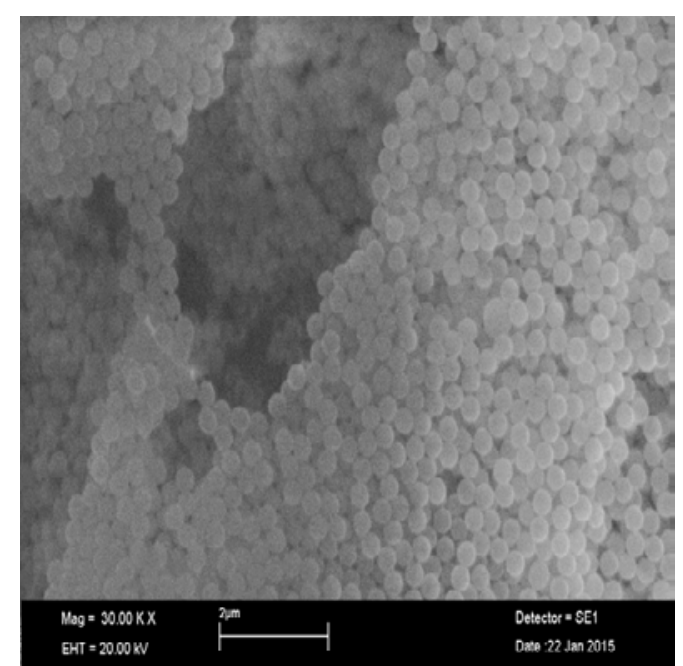

Fig.3: SEM image of vertical deposition via evaporation.

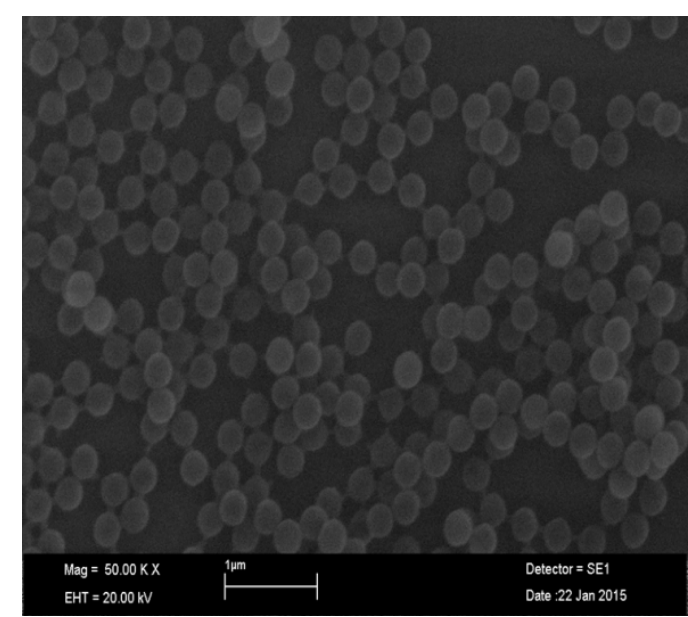

Fig.4: SEM image for floating deposition technique.

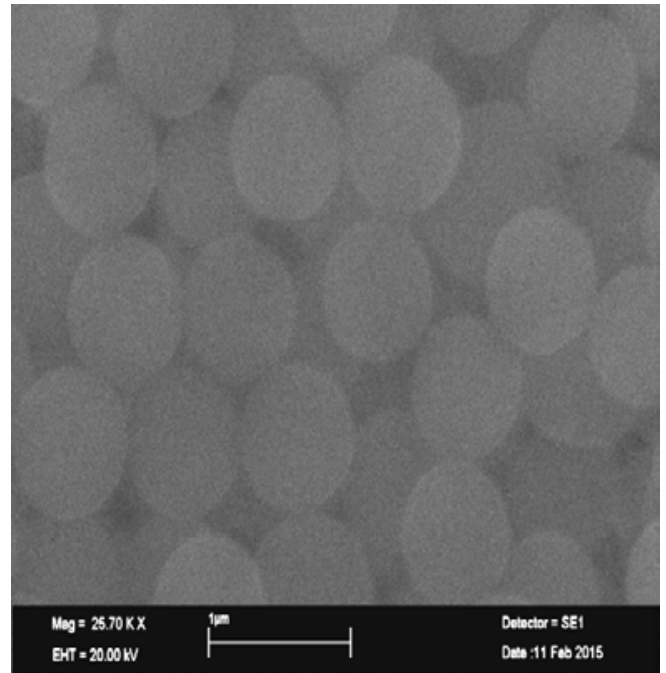

Fig.5: SEM image for spin coating technique.

The PMMA structures tend to be FCC with their (111) planes parallel to the underlying substrate. This configuration is most favored thermo dynamically.

An important phenomenon that can be observed in higher than $85^{\circ} \mathrm{C}$ temperature is the formation of necking (connection between the spheres), which becomes more pronounced as high as in Fig.6. Necking occurs when capillary forces bring the spheres together in the selfassembly process [9]. Necking can be important to increase the mechanical strength of colloidal crystals especially when they are going to be employed as templates for processes such as inverse opal fabrication.

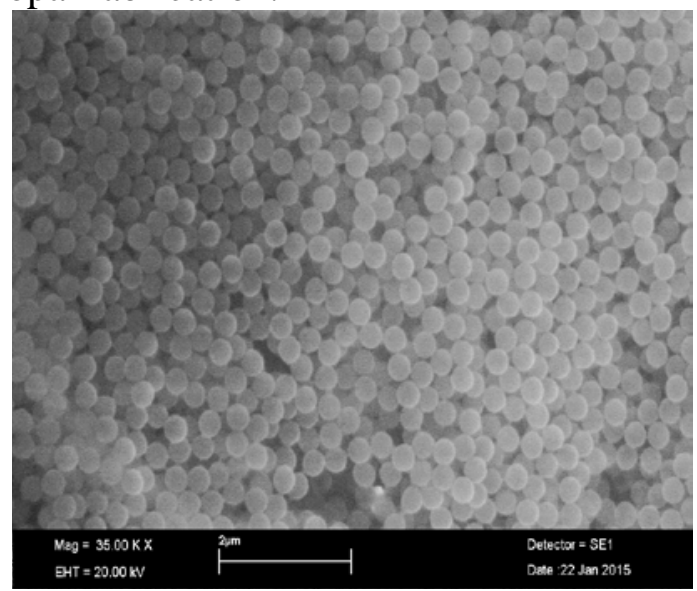

Fig.6: SEM image of necking formation between the spheres. 
Another phenomenon observed in the samples is the change in quality of the colloidal crystals in different areas of the film. The spheres are not completely self-assembled into an ordered colloidal crystal at this region. There is a portion of spheres loosely floating around. This can be attributed to the low concentration of the spheres at the region just below the meniscus contact line. This suggests that a concentration gradient in the vertical deposition self-assembly technique exists. The concentration gradient is due to the gradual evaporation of water inside the beaker which increases the concentration of the spheres in the solution. Therefore more spheres are available at the bottom of the beaker leading to better self-assembly on the bottom region of the glass substrate which is positioned vertically in the beaker. However due to this concentration gradient, the film at the bottom of the glass substrate can be thicker than the top. If the film is too thick, it will eventually shear, crack and peel off the glass substrate. This leaves some bear areas at the bottom of the substrate. The shearing occurs in a vertical direction, which can first be seen as macroscopic cracks on the film prior to detachment. In evaporation induced colloidal crystal formation techniques driven by capillary forces at the meniscus, such as the vertical deposition approach. The vertical deposition technique for colloidal crystal fabrication appears to be a convenient route, but it does not guarantee the formation of large areas of high quality colloidal crystals on the substrate.

SEM image for floating deposition approach technique are shown in Fig.4.

The images clearly show the monolayer (2D) two dimensions PMMA colloidal crystal structure created using this modified floating technique. The size of the modified
PMMA spheres after self-assembly remains constant as the spheres prior self-assembly (383 nm). As seen from the SEM image in Fig.4, large areas of monolayer 2D colloidal crystals are formed without any cracks. A low level of defects and imperfections in the form of separate spheres on top of the monolayer can be detected. Such imperfections may originate from the spheres that are mixed with the water in the Petri dish instead of staying afloat. The role of ethanol is to act as a spreading agent [10]. The mixture of water: ethanol has lower density than pure water $\left(0.911 \mathrm{~g} / \mathrm{cm}^{3}\right.$ compare to 1 $\mathrm{g} / \mathrm{cm}^{3}$ at ambient temperature [11]. It enhances the floating of the PMMA spheres. Also, the addition of ethanol improves the hydrophobic nature of the solvent, which improves the dispersion of the PMMA spheres. The waterethanol mixture has also a higher vapor pressure and evaporation rate than pure water. When the ethanol containing sphere solution is correctly placed onto the water surface, it immediately spreads over it and when the ethanol evaporates, the colloidal spheres stay floating on the water surface leading to effective self-assembly of monolayer colloidal crystal through attractive interactions among the spheres. The modified floating self-assembly approach is considered as a convenient technique, as the whole process occurs in a few minutes at room temperature without optimizing any conditions or needing any special facilities. Large areas of high quality, monolayer PMMA colloidal crystals are produced using this method. Approximately the same discussion was used for spin coating sample.

The optical properties of the colloidal crystal can be described by a modified Bragg's Law of diffraction due to its ordered periodic structure. A combination of Bragg's law of diffraction $(m \lambda=2 d \sin \theta)$ and Snell's 
law of refraction $\left(n_{0} \sin \theta_{0}=n_{1} \sin \theta_{1}\right)$ can be written as [12]:

$$
m \lambda=2 d \sqrt{ } n^{2}-n_{0}^{2} \sin ^{2} \theta
$$

In which $m$ is the order of diffraction, $n_{0}$ and $n$ are the incident refractive index and lattice refractive index respectively, $d$ is the spacing between the planes in the lattice, $\lambda$ is the light wavelength and $\theta$ is the angle between the incident light and the surface normal of the lattice.

For close-packed colloidal crystals with FCC structures, the refractive index $n$ can be taken as an average of refractive indices of the spheres and the interstitial spaces. Eq. (2) shows this relation:

$n_{\text {avg }}=\varnothing n_{\text {sphere }}+(1-\varnothing) n_{\text {voids }}$

where, $\varnothing$ is the solid volume fraction of the structure. Parameter $d$ as the interplanar spacing between $h k l$ indices of Miller planes can be written as:

$d h k l=D \sqrt{ } 2 / \sqrt{ } h^{2}+k^{2}+l^{2}$

As the (111) plane is the predominant plane for the PMMA colloidal crystal,

$d h k l=D \sqrt{ } 2 / 3$

where, $D$ is the average center-tocenter distance between spheres in a close packed lattice that is geometrically identical to the sphere diameter.

For the first order diffraction $(m=1)$ from FCC (111) planes of the PMMA colloidal crystals, Eq. (1) can be written as follows, assuming that $n_{0} \approx 1$ :

$\lambda=1.633 D \sqrt{ } n^{2}{ }_{a v g}-\sin ^{2} \theta$

where, $\lambda$ is the reflectance peak maximum of UV/Vis spectrum measured in $\mathrm{nm}$. For such a structure, this maximum peak corresponds to the position of the photonic band gap (PBG), or stop band where the propagation of light is forbidden. Using the normal incidence of light $\left(\theta=0^{\circ}\right)$ and combining Eq. (2) with (5), $\lambda_{\max }$ can be written as:

$\lambda_{\text {max }}=1.633\left[\varnothing n_{\text {sphere }}+(1-\emptyset) n_{\text {voids }}\right]$

For PMMA colloidal crystals at room temperature, the refractive indices for PMMA and air are $n_{P M M A}=1.492$ and $n_{\text {air }}=1.000$ respectively. The volume fraction of PMMA spheres in the FCC structure is $\emptyset=0.74$ (and thus 0.26 for air). By inserting these values in Eq. (6), the following equation is obtained:

$\lambda_{\max }=1.633 \times 1.364 \times D$

The normal incidence reflectance spectrum of the monolayer PMMA colloidal is obtained using a UV-Vis reflectance spectrometer as in Fig.7.

From this Figure, the reflectance maxima $\left(\lambda_{\max }\right)$ are positioned at 597 $\mathrm{nm}$. The PMMA sphere diameter $(D)$ can now be calculated using Eq. (7): $D=269 \mathrm{~nm}$.

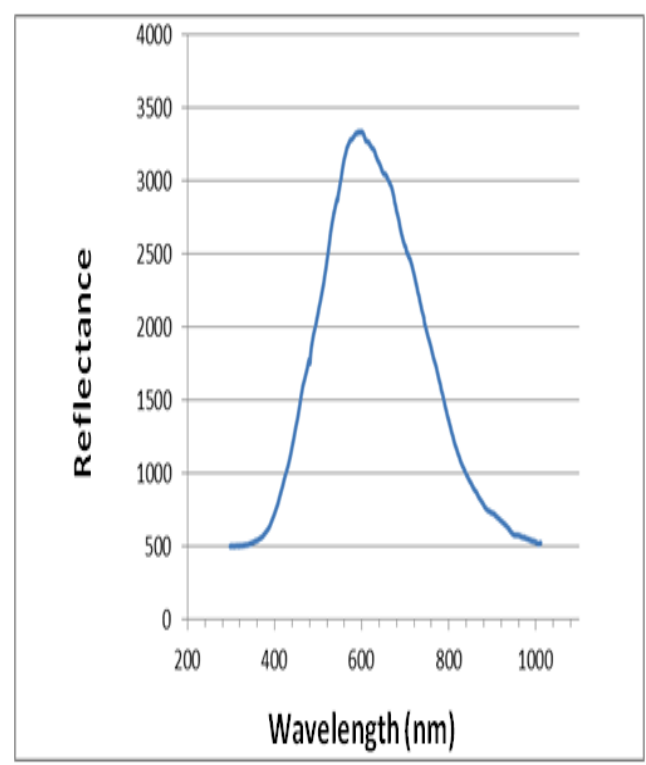

Fig.7: The UV-Vis reflectance spectrum of the monolayer PMMA colloidal. 
This value is $27 \%$ less than the original average sphere diameter determined by the SEM, which is 383 $\mathrm{nm}$. The discrepancy in value may be due to the over simplification of assumptions when using the modified Bragg formula. For instance, the true volume fractions of air and the PMMA sphere can differ from the ideal values of 0.74 and 0.26 , due to crystal defects and size distribution of the PMMA spheres. (A strong agreement was found between the spectral positions of the optical reflections and those predicted by the Bragg diffraction model. The pore size of these materials was found to be directly proportional to the wavelength of the optical stop band).Such defects and dislocations of the spheres may also interfere the diffraction of light and change the lattice constant parameter in the equation. As literature suggests, the modified Bragg equation is not exact because it does not include attenuation of the incident beam and assumes identical contributions from each lattice plane [13]. Although the sphere diameter is not in good accordance with the SEM measurements, the existence of the reflectance maxima $\left(\lambda_{\max }\right)$ confirms the photonic crystal properties of the PMMA colloidal crystal. This technique has been used widely in the literatures [14]. The Bragg reflection seen in the spectrum is attributed to the periodic arrangements of the PMMA colloidal spheres and confirms the high quality of the colloidal crystal produced using the floating technique.

Z-scan results show the change in transmittance of a beam, and the nonlinear refractive index is $n_{2}=9.82787 \times 10^{-12}\left(\mathrm{~cm}^{2} / G W\right)$, while the nonlinear absorption coefficient $\beta=0.04673908(\mathrm{~cm} / \mathrm{GW})$.

Fig.8 the closed aperture Z-scan curve. The change in on-axis intensity is caused by self-focus or self-defocus by the sample as it travels through the beam waist. This modified refractive index distribution then acts like a focusing lens. Hence, when the sample approaches to the focal plane, it will focus the converging beam more tightly.

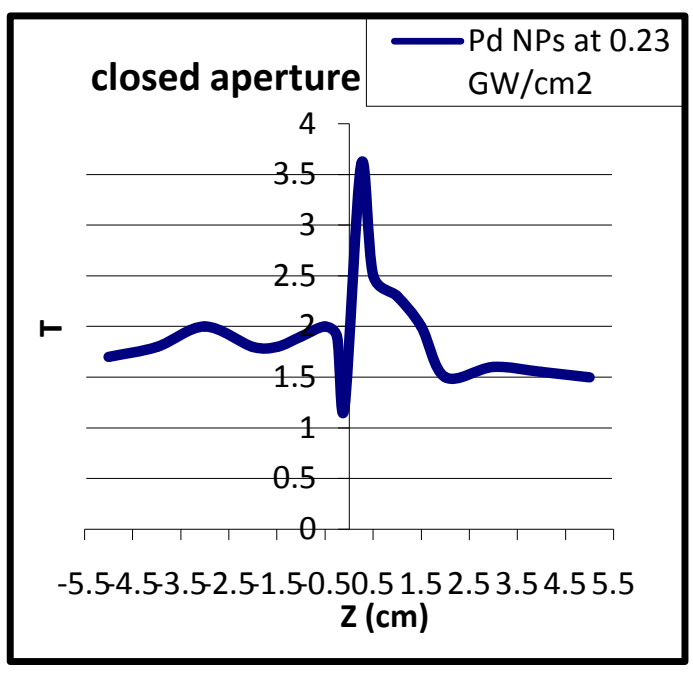

Fig.8: Closed-aperture Z-Scan at $532 \mathrm{~nm}$.

In the far field, this increases beam divergence and is measured as a decrease in energy through the aperture. At the focal plane, the divergence of the beam is unaffected by the sample and the detector measures no net change in transmittance. As it leaves the focal plane, the sample will focus the diverging beam. In the far field, this decreases beam divergence and is measured as an increase in power through the aperture.

The relative on-axis transmittance of the sample measured (at the small aperture of the far-field detector) is given by [15]:-

$$
\mathrm{T}(\mathrm{z}, \Delta \Phi \mathrm{o})=1-\frac{4 \Delta \Phi o \mathrm{Z} / \mathrm{Zo}}{\left[\left(Z^{2} / Z_{r}{ }^{2}\right)+9\right]\left[\left(Z^{2} / Z_{r}^{2}\right)+1\right]}
$$

where $\mathrm{T}$ is the transmittance through the aperture, which is a function of the sample position $\mathrm{Z}, \mathrm{Z}_{\mathrm{r}}$ : is the Rayleigh range, the thickness of the sample is in 
the Rayleigh range. the nonlinear refractive index is calculated from the peak to valley difference of the normalized transmittance by the following formula [15]:-

$n_{2}=\Delta \Phi_{0} / \mathrm{I}_{0} \mathrm{~L}_{\mathrm{eff}} \mathrm{k}$

where,

$\Delta \Phi_{\mathrm{o}}$ :- nonlinear phase shift,

where,

$\mathrm{I}_{0}$ :- Intensity of laser

The intensity of the laser can be shown in Eq. (10)

$\mathrm{I}_{\mathrm{o}}=\mathrm{P} / \mathrm{A}$

$\mathrm{I}_{\mathrm{o}}=978.34\left(\mathrm{GW} / \mathrm{Cm}^{2}\right)$

$\mathrm{P}$ : peak power of the laser $=3 \times 10^{-2} \mathrm{~mJ}$

A:is the area of the laser at the focusing point

$\mathrm{A}=\pi \mathrm{w}_{\mathrm{o}}{ }^{2}=30.66 \times 10^{-4} \mathrm{~cm}^{2}$

where,

$\mathrm{w}_{0}=$ the radius of the laser at the focusing point $=0.003125 \mathrm{~cm}$

$\mathrm{k}=2 \pi / \lambda, \lambda$, is the wavelength of the beam.

$L_{\text {eff }}$ :- the effective length of the sample

Fig.9 shows the behavior of transmittance started linearly at different distances from the far field of the sample position (-Z). The nonlinear refractive index is $\left(9.82787 \times 10^{-12}\right.$ $\left.\left(\mathrm{cm}^{2} / G W\right)\right)$, At the near field the transmittance curve begins to decrease until it reaches the minimum value (Tmin). Afterward, the transmittance begins to increase toward the linear behavior at the far field of the sample position (+Z). The nonlinear absorption coefficient is (0.04673908) $(\mathrm{cm} / \mathrm{GW})$. The change in intensity is caused by two-photon absorption in the sample as it travels through the beam waist.

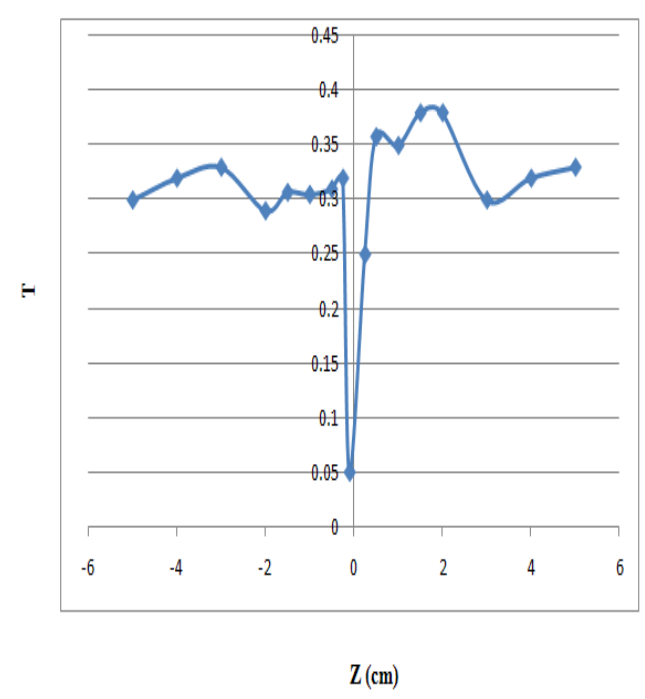

Fig.9: Open-aperture Z-Scan at $532 \mathrm{~nm}$.

\section{Conclusions}

The PMMA colloidal crystals produced in this work with different techniques showed high quality ordered arrays of self-assembled PMMA spheres. Photonic crystal properties of the PMMA colloidal crystals make them good candidates to be utilized not only in templating techniques for production of photonic crystals (inverse opals) and also as opalescent materials for multiple applications, such as displays, sensors, information storage, decoration, camouflage, art and mimicking biological systems. Nonlinear optical properties represented the nonlinear refractive index as well as nonlinear absorption coefficient are due to selffocusing arising from the Kerr effect, and two-photon absorption which appears as minimum transmittance of each of Z-scan trace.

\section{References}

[1] Q. Zhang, G. Cao: Nano Today, 6 (2011) 91. 
[2] A. Stein, B. E. Wilson, S. G. Rudisill, Chem. Soc. Rev, 42 (2013), 2763.

[3] Z. Y. Cai, J. H. Teng, Z. G. Xiong, Y. Q. Li, Q. Li, X. M. Lu, X. S. Zhao, Langmuir, 27 (2011) 5157.

[4] Y. N. Xia, B. Gates, Y. D. Yin, Y. Lu, Adv Mater, 12 (2000) 693.

[5] S. L. Kuai, V. V. Truong, A. Hache, X. F. Hu, J. Appl. Phys., 96 (2004) 5982.

[6] A. Chiappini, C. Armellini, A. Chiasera, M. Ferrari, L. Fortes, M. C. Goncalves, R. Guider, Y. Jestin, R. Retoux, G. N. Conti, S. Pelli, R. M. Almeida, G. C. Righini, J. Non-Cryst Solids, 355 (2009) 1167.

[7] I. Guezguez, A. Karakas, K. Iliopoulos, B. Derkowska-Zielinska, A. El-Ghayoury, A. Ranganathan, P. Batail, A. Migalska-Zalas, B. Sahraoui, M. Karakaya, Opt. Mater, 36 (2014) 106-111.
[8] P.S.P. Silva, Chem. Phys. 428 (2014) 67-74.

[9] G. I. N. Waterhouse and M. R. Waterland, Polyhedron, 26 (2007) 356. [10] L. Group, Max Planck Institute for Polymer Research, Rep. (2012) Mainz, Germany.

[11] T. A. Scott, J. Phys. Chem-Us, 50 (1946) 406.

[12] C. I. Aguirre, E. Reguera, A. Stein, Adv. Funct. Mater, 20 (2010) 2565.

[13] C. Lopez, Adv. Mater, 15 (2003) 1679.

[14] M. Muller, R. Zentel, T. Maka, S. G. Romanov, C. M. S. Torres, Chem Mater, 12 (2000) 2508.

[15] M. Sheik-Bahae, A. A. Said, T. Wel, D. J. Hagan, E. W. Van, IEEE Journal of Quantum Electronic, 26 (1990) 760-769. 